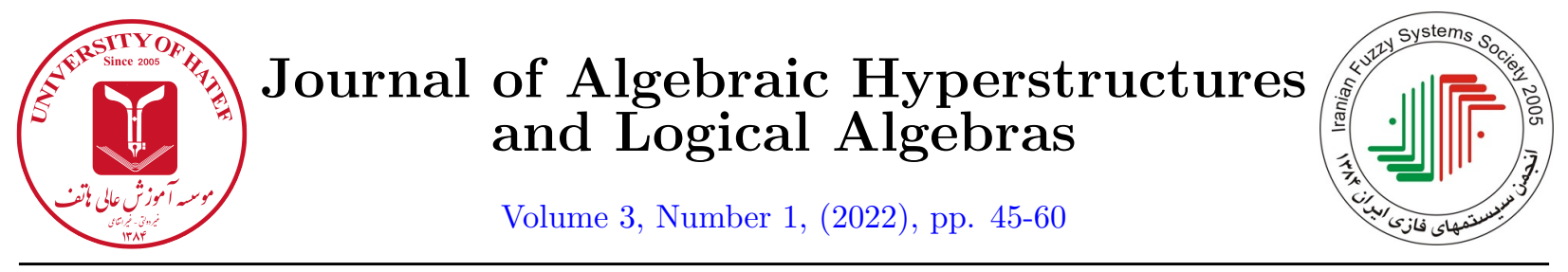

\title{
A longtime season of friendship and scientific collaboration
}

\author{
R. Grigolia ${ }^{1}$ \\ ${ }^{1}$ Tbilisi State University, Department of Mathematics, Georgia \\ revaz.grigolia@tsu.ge
}

"This paper is dedicated to Professor Antonio Di Nola on the occasion of his 75th birthday."

\begin{abstract}
The paper is devoted to a survey of Antonio Di Nola's, and Antonio Di Nola and Revaz Grigolia's scientific researches of longtime scientific collaboration.
\end{abstract}

\section{Article Information}

Corresponding Author:

R. Grigolia;

Received: June 2021;

Accepted: Invited paper;

Paper type: Overview.

\section{Keywords:}

MV-algebra, variety, perfect MV-algebra, category, free, projective, monadic, dynamic.

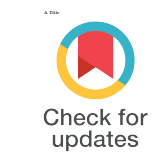

\section{Introduction}

In this issue, we'll give a survey of A. Di Nola's main results and some essential results obtained by A. Di Nola and R. Grigolia concerning Łukasiewicz logic, with its important extensions, and its algebraic models varieties of $M V$-algebras, that are results of the collaboration that it is begun 1992 and continued nowadays. But previously let me give a brief biography of our collaboration and some general historical excursus with key results that have given impact to new deep corollaries.

Our first acquaintance has been in the 14th Linz Seminar on Fuzzy Set Theory 1992, where I have met also P. Belluce and D. Mundici, and we have succeeded quickly to solve a scientific problem. After that, I was invited by A. Di Nola to the Institute of Mathematics of the Faculty of Architecture of the University of Naples in 1993. From now on have begun our long-lasting collaboration. I attended his home in his native town Grumo Nevano, which is a typical Italian small town, where I acquainted with his wife Rosa and Rosa's sister family. Grumo Nevano is a small town where everybody almost knows each other, and I have acquainted with his relatives and I always felt a very warm attitude from them. Then A. Di Nola was invited to the Symposium on Language, Logic, and Computation that took place in the Georgian mountain resort Gudauri (1995), where Antonio has acquainted with L. Esakia (head of Georgian logic group) and other Georgian logicians, and, I would like to notice, a warm party in Esakia's home in Tbilisi (capital of

https://doi.org/10.52547/HATEF.JAHLA.3.1.5 
Georgia). Notice, that he attended Tbilisi and my home many times. The conference "Ordered Groups and Lattices in Algebraic Logic" (OGLAL2011), organized by Salerno and Tbilisi Universities, Kurt Gödel Society, Studia Logica, have been held in Tbilisi, dedicated to the living memory of great Georgian logician Prof. Leo Esakia, was part of the regular series Order and Algebra in Logic, started in Napoli in 1991 (Order in Algebra and Logic with application, 1991, University of Napoli, Italy).

It would be noticed that our collaboration carries interactive character - sometimes the idea arises from Antonio, sometimes from me but we work together with posed problem. Our collaboration was continued with the University of Salerno, where Antonio became a professor of the Department of Mathematics, and Tbilisi State University that was strengthened by a special scientific agreement. According to the agreement it was held two scientific conferences in Salerno (2014) and Tbilisi (2015). I would like also to notice that over a long period of our collaboration I have acquainted with our other co-authors: Peter Belluce, Ada Lettieri, who suddenly passed away, unfortunately, Giovani Panti, Luca Spada, Giacomo Lenzi, Gaetano Vitale, Vincenzo Loia.

Many-valued logic is the complex of studies that originated from the papers of Eukasiewicz [32, 33] and Post [37] in the twenties of the last century. It should be noted that these logics are defined axiomatically. From the logical point of view, I give preference to Eukasiewicz logic since Łukasiewicz logic has sensible interpretation and, moreover, philosophical hidden. Łukasiewicz logic and classical logic have the same logical connectives, but different interpretations that emphasize their difference.

Taking into account Eukasiewicz's idea on infinite valued logic afterward C. C. Chang has been developed its algebraic counterpart - the variety of $M V$-algebras [7] (1958), and proved the completeness theorem for Eukasiewicz logic with respect to the variety $\mathbf{M V}$ of $M V$-algebras. From this point, it is begun developing the theory of $M V$-algebras. C.C. Chang constructed $\Gamma$ functor from the category of linearly ordered groups with strong unit to the category of linearly ordered $M V$-algebras [ 8$]$ (1959), and then D. Mundici generalized this construction to all lattice ordered groups with strong unit, and, moreover, $\Gamma$ established a natural equivalence (i.e., a full, faithful, dense functor) between the category of lattice ordered groups with strong unit and the category of all $M V$-algebras [35] (1963).

Di Nola's contribution to the study of $M V$-algebras, witnessed by his work [10] (1991) that has citations in the fundamental monograph [9] (2000), includes: a functional representation theorem for all $M V$-algebras (also known as Di Nolas Representation Theorem).

Subvarieties of $M V$-algebras have been studied by R. Grigolia [2.9] (1973), Y. Komori [30] (1981), A. Di Nola and A. Lettieri [24] (1999). It is known that any such variety is generated by finitely many algebras, and explicit axiomatizations have been obtained. R. Grigolia axiomatized all finitely valued Łukasiewicz logics $L_{n}\left(1 \leq n \in Z^{+}\right)$and corresponding to them varieties $\mathbf{M V}_{\mathbf{n}}$ generated by finite chain $M V$-algebras (1973). Y. Komori described all subvarieties of the variety MV (1981). A. Di Nola and A. Lettieri axiomatized all subvarieties of the variety MV (1999).

There are $M V$-algebras which are not semisimple, i.e. the intersection of their maximal ideals (the radical of A) is different from $\{0\}$. Non-zero elements from the radical of A are called infinitesimals. It is worth stressing that the existence of infinitesimals in some $M V$-algebras is due to the remarkable difference of behavior between Boolean algebras and $M V$-algebras.

Perfect $M V$-algebras, that were introduced by B. Belluce, A. Di Nola, and A. Lettieri in [4] (1993), are those $M V$-algebras generated by their infinitesimal elements or, equivalently, generated by their radical [3] (2007). They generate the smallest non locally finite subvariety of the variety $\mathbf{M V}$ of all $M V$-algebras. An important example of a perfect $M V$-algebra is the subalgebra $S$ of the Lindenbaum algebra $L$ of first order Łukasiewicz logic generated by the classes of formulas which are valid when interpreted in $[0,1]$ but non-provable. Hence perfect $M V$-algebras are directly connected with the very important phenomenon of incompleteness in Łukasiewicz first order logic (see [2, 40]. One of the interesting results established by A. Di Nola and A. Lettieri is a categorical equivalence between abelian $\ell$-groups and perfect $M V$-algebras [2:3] (1994). Infinitesimal elements of perfect $M V$-algebra spring to mind the idea of quasi-false and quasi-truth. Following this idea, A. Di Nola, R. Grigolia, and E. Turunen have been published the monograph Fuzzy Logic of Quasi-Truth: An Algebraic Treatment [21] (2016).

Free $M V$-algebras, which is an important object of a variety of algebras, have been studied by Di Nola, R. Grigolia and G. Panti [1.9] (1998) and by G. Panti [36] (1999). Projective $M V$-algebras, which play important role in unification problem, have been studied by Di Nola, R. Grigolia [II] (2003), Di Nola, R. Grigolia and A, Lettieri [15] (2008), and it should be also noticed L. M. Cabrer and D. Mundici [6]. 
Let $E$ be an equational theory. The $E$-unification problem is: given two terms $s, t$ (built from function symbols and variables), to find a unifier for them, that is, a uniform replacement of the variables occurring in $s$ and $t$ by other terms that make $s$ and $t$ equal by modulo $E$.

Dzik's main result [25] entails as a special case that each subvariety of $M V$-algebras generated by a single finite chain has a unitary unification type. V. Marra and L. Spada have proved that the unification type of $M V$-algebras is nullary and that nullary unification problems already occur over two variables [34].

The variety generated by perfect $M V$-algebras has been investigated by A. Di Nola, R. Grigolia and G. Lenzi in [14] (2016). It is shown that for $m$-generated algebras from this variety to be finitely presented is equivalent to be projective. The variety generated by perfect algebras has a unitary unification type and it is shown that the logic corresponding to this variety is structurally complete.

Monadic $M V$-algebras (monadic Chang algebras by Rutledges terminology) were introduced and studied by Rutledge in [39] (1959), using a functional approach, as an algebraic model for the predicate calculus of Łukasiewicz infinite-valued logic, in which only a single individual variable occurs. Rutledge followed P.R. Halmos study of monadic Boolean algebras. In view of the incompleteness of the predicate calculus, the result of Rutledge in [3:] , showing the completeness of the monadic predicate calculus, has been of great interest.

After 44 years, extending the signature of $M V$-algebra by unary monadic (modal) operation, we (A. Di Nola and R. Grigolia) in [12] (2004) defined and studied monadic $M V$-algebras as pairs of $M V$-algebras one of which is a special case of relatively complete subalgebra named $m$-relatively complete. An $m$-relatively complete subalgebra determines a unique monadic operator. A necessary and sufficient condition is given for a subalgebra to be $m$-relatively complete. After this scientific work serial works appeared by many authors devoted to various types of monadic algebras.

We introduced a new logic [I7] (2015), a multimodal epistemic Łukasiewicz logic, which is an extension of the infinitely valued Łukasiewicz logic, the language of the logic is extended by unary connectives that are interpreted as modal operators (knowledge operators). We proposed the use of such logic in studying immune system.

Following K. Segerberg [4T] (1977), D. Kozen [3] (1979) and V. Pratt [38] (1980), who have been introduced dynamic (classical) propositional logic, that is a formal system for reasoning about programs, and dynamic algebras, dynamic propositional Łukasiewicz logic $D P L$ (dynamic $n$-valued propositional Eukasiewicz logic $\left.D P L_{n}\right)$ and dynamic $M V$-algebras (dynamic $M V_{n}$-algebras) are introduced and theories of the logic $D P L\left(D P L_{n}\right)$ and dynamic $M V$-algebras $\left(M V_{n}\right.$-algebras) are developed [22] (2020). Dynamic $M V$-algebras (dynamic $M V_{n}$-algebras) are algebraic counterparts of the logic $D P L\left(D P L_{n}\right)$, that in turn represent two-sorted algebras that combine the varieties of $M V$-algebras ( $M V_{n}$-algebras) and regular algebras into a single finitely axiomatized variety resembling $R$-module with scalar multiplication. The results obtained for dynamic Łukasiewicz logic have been applied for the immune system in [18] (2021) having another interpretation of modal operators

\section{The variety of $M V$-algebras and its subvarieties}

An algebra $A=(A, 0, \neg, \oplus)$ with one binary and one unary and one nullary operations is a $M V$-algebras if it satisfies:

MV1. $(A, 0, \oplus)$ is an abelian monoid

MV2. $\neg \neg x=x$

MV2. $x \oplus \neg 0=\neg 0$

MV3. $y \oplus \neg(y \oplus \neg x)=x \oplus \neg(x \oplus y)$.

We set $1=\neg 0$ and $x \odot y=\neg(\neg x \oplus \neg y)$. We shall write $a b$ for $a \odot b$ and $a^{n}$ for $\underbrace{a \odot \cdots \odot a}_{n \text { times }}$, for given

$a, b \in A$. Every $M V$-algebra has an underlying ordered structure defined by $x \leq y$ iff $\neg x \oplus y=1$. Then $(A ; \leq, 0,1)$ is a bounded distributive lattice. Moreover, the following property holds in any $M V$-algebra:

$$
x y \leq x \wedge y \leq x \vee y \leq x \oplus y .
$$

The unit interval of real numbers $[0,1]$ endowed with the following operations: $x \oplus y=\min (1, x+y), x \odot$ 
$y=\max (0, x+y-1), \neg x=1-x$, becomes an $M V$-algebra. It is well known that the $M V$-algebra $S=([0,1], \oplus, \odot, \neg, 0,1)$ generate the variety $\mathbf{M V}$ of all $M V$-algebras, i. e. $\mathcal{V}(S)=\mathbf{M V}$.

$M V$-algebras are algebraic models of Łukasiewicz logic $\mathrm{E}$ [7]. The axioms of the Eukasiewicz logic are the following [3.3]:

(L1) $\varphi \rightarrow(\psi \rightarrow \varphi)$,

(L2) $(\varphi \rightarrow \psi) \rightarrow((\psi \rightarrow \chi) \rightarrow(\varphi \rightarrow \chi))$,

(L3) $(\neg \varphi \rightarrow \neg \psi) \rightarrow(\psi \rightarrow \varphi)$,

(L4) $((\varphi \rightarrow \psi) \rightarrow \psi) \rightarrow(\psi \rightarrow \varphi) \rightarrow \varphi)$.

$M V_{n}$-algebras, that was introduced by R. Grigolia in [29] (1973), are algebraic models of $n$-valued Eukasiewicz logic $\mathrm{E}_{n}$. The axioms of the logic $\mathrm{L}_{n}$, that was given by R. Grigolia [29] (1973), are the Łukasiewicz logic plus the following:

$\left(L_{n} 5\right) \varphi^{n} \leftrightarrow \varphi^{n-1}$,

$\left(L_{n} 6\right) n\left(\varphi^{k}\right) \leftrightarrow\left(k\left(\varphi^{k-1}\right)\right)^{n}$,

for every integer $2 \leq k \leq n-2$ that does not divide $n-1$.

An $M V$-algebra $A=(A, \oplus, \odot, \neg, 0,1)$ is $M V_{n}$-algebra if it satisfies the identities: $x^{n}=x^{n-1},\left(n\left(x^{k}\right)=\right.$ $\left(k\left(x^{k-1}\right)\right)^{n}$ for every integer $2 \leq k \leq n-2$ that does not divide $n-1$ [29] (1973).

The subvariety $\left.\mathbf{M V}_{n}\right)\left(=\mathcal{V}\left(S_{n}\right)\right)$ of $\mathbf{M V}$ (also named by Grigolia's subvariety [I]) is generated by $S_{n}=(\{0,1 / n, \ldots, n-1 / n, n\}, \oplus, \odot, \neg, 0,1)$.

Chang discovered a correspondence between totally ordered (= linearly ordered) $M V$-algebras and totally ordered abelian groups [7]. Mundici, in [35], extended this correspondence to a $\Gamma$ functor between $M V$-algebras and lattice-ordered abelian groups (abelian $\ell$-groups) $(G,+,-, 0, u)$ with strong unit $u$, and proved that $\Gamma$ is a categorical equivalence. For every abelian $\ell$-group $G$, the functor $\Gamma$ equips the unit interval $[0, u]$ with the operations:

$$
\begin{aligned}
& x \oplus y=u \wedge(x+y) ; \\
& x \odot y=0 \vee(x+y-u) ; \\
& \neg x=u-x ; 0=0 ; \\
& 1=u .
\end{aligned}
$$

It is easy to see that the resulting structure $([0, u], 0, \neg, \oplus)$ is an $M V$-algebra.

Following Komori [30], set $S_{n}^{\omega}=\Gamma\left(\left(Z \times_{l e x} Z\right),(n, 0)\right)$, where $Z$ is the totally ordered additive group of integers, and $Z \times_{l e x} Z$ is the lexicographic product of $Z$ by itself; and let $S_{n}=\Gamma(Z, n)\left(\in \mathbf{M V}_{\mathbf{n}}\right)$; note that $S_{n}$ is a subalgebra of $S_{n}^{\omega}$.

In [30], Komori proved that every subvariety $\mathbf{V}$ of $\mathbf{M V}$ is of the form $\mathbf{V}=\mathcal{V}\left\{S_{m_{1}}, \ldots, S_{m_{r}}, S_{t_{1}}^{\omega}, \ldots, S_{t_{s}}^{\omega}\right\}$ for some finite sets $I=\left\{m_{1}, \ldots, m_{r}\right\}$ and $J=\left\{t_{1}, \ldots, t_{s}\right\}$, not both empty.

Let $\mathcal{V}\left(A_{1}, \ldots, A_{n}\right)$ denotes the subvariety of $\mathbf{M V}$ which is generated by the $M V$-algebras $A_{1}, \ldots, A_{n}$. For every $i \in Z^{+}$let

$$
\delta(i)=\{n \in Z: 1 \leq n \text { and } n \text { is a divisor of } i\} .
$$

Furthermore, if $J$ is a nonempty finite subset of $Z^{+}$and $i=2,3,4, \ldots$ let $\Delta(i, J)=\left\{d \in \delta(i)-\bigcup_{j \in J} \delta(j)\right\}$. In case $J=\emptyset$ we define $\Delta(i, \emptyset)=\delta(i)$.

\section{Di Nola - Lettieri Assertion}

Let $\mathbf{V}$ be a proper subvariety of $\mathbf{M V}$. Then there exist finite sets $I$ and $J$ of positive integers with $I \cup J \neq \emptyset$, such that for any $M V$-algebra $A$ we have $A \in \mathbf{V}$ iff $A$ satisfies the equations

$$
\begin{gathered}
\left((n+1) x^{n}\right)^{2}=2 x^{n+1}, \text { where } n=\max \{I \cup J\} ; \\
\left(p x^{p-1}\right)^{n+1}=(n+1) x^{p},
\end{gathered}
$$

for every positive integer $1<p<n$ such that $p$ is not a divisor of any $i \in I \cup J$;

$$
(n+1) x^{q}=(n+2) x^{q}, \text { for every } q \in \bigcup_{j \in J} \Delta(i, J) .
$$


Equations (1) and (2) tackle subvarieties with no finite algebras as generators. Equations (3) are added to deal with subvarieties having mixed finite and infinite generators.

From this A. Di Nola and A. Lettieri result, we can conclude that any subvariety of MV is finitely axiomatizable.

\section{Di Nola's representation theorem}

Let $I$ be a non-empty set. Let $(P(I), \cup, \cap, I, \emptyset, I)$ be the Boolean algebra of all subsets of $I$. A subset $F \subset P(I)$ is said to be filter if: 1) $I \in F, 2)$ if $X, Y \in F$ then $X \cap Y \in F, 3)$ if $X \in F$ and $X \subset Y$ then $Y \in F$. A filter $F$ is proper if $F \neq P(I)$. A proper filter $U$ is called ultrafilter if it is a maximal proper filter with respect to the inclusion between filters.

Let $\left(A_{i}\right)_{i \in I}$ be a nonempty indexed family of algebras of the same type, and suppose $F$ is a filter over I. We define the binary relation $\theta_{F}$ on $\prod_{i \in I} A_{i}$ by $(a, b) \in \theta_{F}$ iff $\{i \in I: a(i)=b(i)\} \in F$ which is a congruence relation.

Given a nonempty indexed family of algebras $\left(A_{i}\right)_{i \in I}$ of the same type and a proper filter $F$ over $I$, define the reduced product $\prod_{i \in I} A_{i} / F$ as follows. Let its universe $\prod_{i \in I} A_{i} / F$ be the set $\prod_{i \in I} A_{i} / \theta_{F}$, and let $a / F$ denote the element $a / \theta_{F}$. For an $n$-ary function symbol $f$ and for $a_{1}, \ldots, a_{n} \in \prod_{i \in I} A_{i}$, let

$$
f\left(a_{1} / F, \ldots, a_{n} / F\right)=f\left(a_{1}, \ldots, a_{n}\right) / F .
$$

A reduced product $\prod_{i \in I} A_{i} / U$ is called an ultraproduct if $U$ is an ultrafilter over $I$. If all the $A_{i}=A$, then we write $A^{I} / U$ and call it an ultrapower of $A$.

Proposition 3.1. (Di Nola's representation theorem) [10]. Up to isomorphism, every MV-algebra $A$ is an algebra of $[0,1]^{I} / U$-valued functions over some set only depending on the cardinality of $A$.

\section{Perfect $M V$-algebras}

The class of perfect $M V$-algebras is a full subcategory of the category of $M V$-algebras. In general, there are $M V$-algebras which are not semisimple. Roughly speaking we can say that a non-semisimple $M V$-algebra $A$ has a non-zero radical. We call a non-zero element from the radical of $A$ an infinitesimal. The first example of a non-simple $M V$-chain was given by Chang in []], where the $M V$-algebra $C$ is described.

Chang's $M V$-algebra $C$ [I]], which is our main interest, is defined on the set

$$
C=\{0, c, \ldots, n c, \ldots, 1-n c, \ldots, 1-c, 1\}
$$

by the following operations (consider $0=0 c$ ): $x \oplus y=$

- $(m+n)) c$ if $x=n c$ and $y=m c$;

- $1-(m-n) c$ if $x=1-n c$ and $y=m c$ and $0<n<m$;

- $1-(n-m) c$ if $x=n c$ and $y=1-m c$ and $0<m<n$;

- 1 otherwise;

$\neg x=1-n c$ if $x=n c, \neg x=n c$ if $x=1-n c$.

The $M V$-algebra $C$ is isomorphic to the algebra $S_{1}^{\omega}$ defined by Komori in [30].

The algebra $C$ has remarkable properties:

(1) $C$ is generated by its radical

(2) $C=\operatorname{Rad}(C) \cup \neg \operatorname{Rad}(C)$

(3) $C / \operatorname{Rad}(C) \cong\{0,1\}$. 
Hence $C$ is just made by infinitesimal elements and co-infinitesimal elements. We then would like to describe a class of $M V$-algebras containing $C$ and whose elements share the above properties. Then we can think of such a class as the one made by $M V$-algebras which are, up to infinitesimal elements, like the 2-elements Boolean algebra $\{0,1\}$.

We say that an $M V$-algebra $A$ is perfect if for each element $x \in A, \operatorname{ord}(x)<\infty$ iff $\operatorname{ord}(\neg x)=\infty$, where the order of an element $x$, in symbols ord $(x)$, is the least integer $m$ such that $m x=1$; if no such integer $m$ exists then $\operatorname{ord}(x)=\infty$.

An ideal of an $M V$-algebra $A$ is a subset $J$ of $A$ satisfying the following conditions:

J1) $0 \in J$,

J2) If $x \in J, y \in A$ and $y \leq x$ then $y \in J$,

J3) If $x \in J$ and $y \in J$ then $x \oplus y \in J$.

We say that an ideal $J$ of an $M V$-algebra $A$ is perfect if for every $x \in A$, there is an $n \in \mathbb{N}$ such that $x^{n} \in J$ iff $(\neg x)^{m} \notin J$ for all $m \in \mathbb{N}$.

The intersection of any family of ideals of $A$ is an ideal of $A$. For every subset $W \subseteq A$, the intersection of all ideals $J \supseteq W$ is said to be the ideal generated by $W$. In particular, given an element $z$ of an $M V$-algebra $A$, the ideal $\langle z\rangle$ generated by the singleton $\{z\}$ is called the principal ideal generated by $z$, and we have $\langle z\rangle=\{x \in A: n z \geq x\}$ for some integer $n \geq 0$.

Filter and perfect filter are defined dually.

Let $X$ be a subset of the algebra $A$. We say that the algebra $A$ is generated by the set $X$ if $A$ coincides with the intersectionof all subalgebras containung the set $X$ : in notation $A=\langle X\rangle$.

Proposition 4.1. [2]] (i) Let $A$ be a perfect $M V$-algebra. Then $\operatorname{Rad}(A)$ is a unique maximal ideal of $A$; (ii) An ideal $J \subseteq A$ is perfect iff $A / J$ is perfect;

(iii) Let $A$ be an $M V$-algebra, the following statements are equivalent:

(1) A is perfect;

(2) Every ideal $J \subseteq A$ is perfect;

(iv) In an $M V$-algebra $A$, the algebra $\langle\operatorname{Rad}(A)\rangle$ generated by $\operatorname{Rad}(A)$ is a perfect subalgebra of $A$;

(v) Let $A$ be a perfect $M V$-algebra. Then $A=\langle\operatorname{Rad}(A)\rangle$;

(vi) Let $A$ be an $M V$-algebra. Then the following are equivalent:

(1) $A$ is perfect.

(2) $A / \operatorname{Rad}(A)=\{0,1\}$.

(vii) Let $A$ be a perfect $M V$-algebra and $f$ a homomorphism. Then $f(A)$ is a perfect $M V$-algebra;

(viii) Let $A$ be a non semisimple $M V$-algebra. Then A contains a copy of $C$ as a subalgebra;

\subsection{The category of Perfect $M V$-algebras}

A relevant the fact concerning perfect $M V$-algebras is that each one of them is generated by its infinitesimals. This turns out to induce a very special structure on the generated algebra. Perfect $M V$-algebras can be seen as an extreme case of non-archimedean $M V$-algebras. Thus, the role of perfect $M V$-algebras is important because it is strictly linked with the role of infinitesimals. An important example of a perfect $M V$-algebra can be found as a subalgebra $S$ of the Lindenbaum algebra $L$ of the first order Łukasiewicz logic. Hence perfect $M V$-algebras are directly connected with a very important phenomenon of the first order Łukasiewicz logic, namely, with the incompleteness of such a logic.

Perfect $M V$-algebras form a full subcategory of the category of all $M V$-algebras. We denote the category of perfect $M V$-algebras by Perfect.

Proposition 4.2. [2.3, [2]] (i) The category Perfect of perfect $M V$-algebras is equivalent to the category of abelian l-groups;

(ii) $\{0,1\}$ is a terminal and initial object of Perfect;

(iii) Perfect has pull-backs;

(iv) Perfect has arbitrary products;

(v) Perfect has the amalgamation property. 


\subsection{The variety generated by Perfect $M V$-algebras}

It is worth remarking that the class of perfect algebras does not form a variety, so the problem of studying the proper subvariety of the variety of all $M V$-algebras generated by all perfect $M V$-algebras arises.

Let $\mathcal{V}(\operatorname{Per} f)$ be the variety generated by all perfect algebras, and $\mathcal{V}(C)$ be the variety generated by Chang's algebra $C$. Then the following theorem holds:

Proposition 4.3. [2.3, 21] $(i)$ An $M V$-algebra $A$ is in the variety $V(C)$ iff $A$ satisfies the identity:

$$
(x \oplus x) \odot(x \oplus x)=(x \odot x) \oplus(x \odot x) ;
$$

(ii) $\mathcal{V}(C)=\mathcal{V}(\operatorname{Per} f)$;

(iii) Let $A$ be a perfect non-Boolean $M V$-chain. Then $\mathcal{V}(A)=\mathcal{V}(\operatorname{Per} f)$;

(iv) Let $A \in \mathcal{V}(C)$. Then $A$ is a subdirect product of perfect $M V$-chains.

Proposition 4.4. [[]] $\mathcal{V}(C)=\mathcal{Q} \mathcal{V}(C)$, where $\mathcal{Q} \mathcal{V}(C)$ is a quasivariety generated by $C$.

Since $\mathcal{Q} \mathcal{V}\left(F_{\mathrm{MV}}(\omega)\right)=\mathcal{Q} \mathcal{V}(C)$, from this proposition we obtain directly

Corollary 4.5. The logic $L_{P}$, corresponding to the variety of perfect $M V$-algebras, is structurally complete.

\section{$5 \quad$ Free and projective algebras}

Let $\mathbf{V}$ be a variety. An algebra $A \in \mathbf{V}$ is said to be a free algebra over $\mathbf{V}$, if there exists a set $A_{0} \subset A$ such that $A_{0}$ generates $A$ and every mapping $f$ from $A_{0}$ to any algebra $B \in \mathbf{V}$ is extended to a homomorphism $h$ from $A$ to $B$. In this case, $A_{0}$ is said to be the set of free generators of $A$. If the set of free generators is finite, then $A$ is said to be a free algebra of finitely many generators. We denote a free algebra $A$ with $m \in(\omega+1)$ free generators by $F_{\mathbf{V}}(m)$. We shall omit the subscript $\mathbf{V}$ if the variety $\mathbf{V}$ is known. We can also define the $m$-generate free algebra $A$ on the generators $g_{1}, \ldots, g_{m}$ over the variety $\mathbf{K}$ in the following way: the algebra $A$ is a free algebra on the generators $g_{1}, \ldots, g_{m}$ iff for any $m$ variable identity $p\left(x_{1}, \ldots, x_{m}\right)=q\left(x_{1}, \ldots, x_{m}\right)$, the identity holds in the variety $\mathbf{K}$ iff the equation $p\left(g_{1}, \ldots, g_{m}\right)=q\left(g_{1}, \ldots, g_{m}\right)$ is true in the algebra $A$ on the generators [5].

Let $\mathbf{V}$ be any variety of algebras. An algebra $A$ is said to be retract of the algebra $B$, if there are homomorphisms $\varepsilon: A \rightarrow B$ and $h: B \rightarrow A$ such that $h \varepsilon=I d_{A}$, where $I d_{A}$ is the identity map over $A$. An algebra $A \in \mathbf{V}$ is called projective, if for any $B, C \in \mathbf{V}$, any onto homomorphism $\gamma: B \rightarrow C$ and any homomorphism $\beta: A \rightarrow C$, there exists a homomorphism $\alpha: A \rightarrow B$ such that $\gamma \alpha=\beta$. Notice that in varieties, projective algebras are characterized as retracts of free algebras.

A subalgebra $A$ of $F_{\mathbf{V}}(m)$ is said to be projective subalgebra if there exists an endomorphism $h: F_{\mathbf{V}}(m) \rightarrow$ $F_{\mathbf{V}}(m)$ such that $h\left(F_{\mathbf{V}}(m)\right)=A$ and $h(x)=x$ for every $x \in A$.

Let $F_{\mathbf{V}_{n}}(m)$ be $m$-generated free $M V$-algebra in the variety

$$
\mathbf{V}_{n}=\mathcal{V}\left(\left\{S_{1}, \ldots, S_{n}\right\}\right)
$$

Let $g_{1}^{(n)}, \ldots, g_{m}^{(n)} \in F_{\mathbf{V}_{n}}(m)$ be free generators of $F_{\mathbf{V}_{n}}(m)$.

On $Z^{+}$we define the function $v_{m}(x)$ as follows: $v_{m}(1)=2^{m}, v_{m}(2)=3^{m}-2^{m}, \ldots, v_{m}(n)=(n+1)^{m}-$ $\left(v_{m} n_{1}+\ldots v_{m}\left(n_{k-1}\right)\right)$, where $n_{1}(=1), \ldots, n_{k-1}$ are all the divisors of $n$ distinct from $n\left(=n_{k}\right)$.

Proposition 5.1. [1.9] $F_{\mathbf{V}_{n}}(m) \cong S_{1}^{v_{m}(1)} \times \ldots \times S_{n}^{v_{m}(n)}$.

Let $F_{\mathbf{M V}}(m)$ be $m$-generated free $M V$-algebra in the variety $\mathbf{M V}$.

Proposition 5.2. [I]] $(i) F_{\mathbf{M V}}(m)$ is isomorphic to a subalgebra of an inverse limit $F_{\infty}(m)$ of a chain of order type $\omega^{*}$ of finite algebras, for $m \in \omega$, and the finite algebras are isomorphic to $F_{\mathbf{V}_{n}}(m)$.

(ii) The subalgebra $\left.F_{\mathbf{M V}}(m)\right)$ of the direct product $\prod_{n=1}^{\infty} F_{\mathbf{V}_{n}}(m)$ generated by $z_{i}=\left(g_{i}^{(1)}, g_{i}^{(2)}, \ldots\right) \in \prod_{n=1}^{\infty} F_{\mathbf{V}_{n}}(m)$ is a free $M V$-algebra with the free generators $z_{1}, \ldots, z_{m}$, where $i=1, \ldots, m$, and are free generators of the free $m$-generated $M V$-algebra $F_{\mathbf{V}_{n}}(m)$.

(iii) The free $M V$-algebra $F_{\mathbf{M V}}(\omega)$ with countably many free generators is isomorphic to a subalgebra of the inverse limit $F_{\infty}(\omega)$ of the inverse system $\left\{F_{\mathbf{V}_{i}}(\omega), \pi_{i j}\right\}$, where $F_{\mathbf{V}_{i}}(\omega)$ is the free MV-algebra with countably many free generators in the variety $\mathbf{V}_{i}$. 
In addition, it would be noticed, that in [20] the $n$-generated free $M V$-algebra is represented as a quotient of the disjoint union of all the $n$-generated free $V_{n}$-algebras. Such a quotient can be seen as the direct limit of a system consisting of all free $V_{n}$-algebras and special maps between them as morphisms.

Proposition 5.3. [II] Let $\mathbf{K}$ be any variety of algebras and $F_{\mathbf{K}}(m)$ be the m-generated free algebra of a variety $\mathbf{K}$ and $g_{1}, \ldots, g_{m}$ be its free generators. Then the $m$ generated subalgebra $A$ of $F_{\mathbf{K}}(m)$ with the generators $a_{1}, \ldots, a_{m} \in A$ is projective if and only if there exist polynomials $P_{1}\left(x_{1}, \ldots, x_{m}\right), \ldots, P_{m}\left(x_{1}, \ldots, x_{m}\right)$ such that

$$
P_{i}\left(g_{1}, \ldots, g_{m}\right)=a_{i}
$$

and

$$
P_{i}\left(P_{1}\left(x_{1}, \ldots, x_{m}\right), \ldots, P_{m}\left(x_{1}, \ldots, x_{m}\right)\right)=P_{i}\left(x_{1}, \ldots, x_{m}\right)
$$

$i=1, \ldots, m$.

Proposition 5.4. [II] (1) Let $A$ be finite $M V$-algebra of a locally finite subvariety $\mathbf{V}_{n}$ of the variety $\mathbf{M V}$ of all $M V$-algebras. Then the following statements are mutually equivalent:

(i) $A$ is projective in $\mathbf{V}_{n}$,

(ii) $A$ is isomorphic to $S_{1} \times A^{\prime}$ for some finite $M V$-algebra $A^{\prime}$.

(2) Any subalgebra of the m-generated free algebra $F_{\mathbf{V}_{n}}(m)$ is projective $M V$-algebra in the variety $\mathbf{V}_{n}$.

(3) Any endomorphic image of $F_{\mathbf{V}_{n}}(m)$ is an m-generated projective $M V$-algebra in $\mathbf{V}_{n}$.

(4) If $A$ is finite projective $M V$-algebra in the variety $\mathbf{M V}$ then $A$ is isomorphic to $S_{1}$.

Let $S_{1}^{\omega(1)}=\Gamma\left(Z \times_{\text {lex }} Z,(1,0)\right)=C, S_{1}^{\omega(m)}=\Gamma(\underbrace{Z \times_{\text {lex }} \ldots \times_{\text {lex }} Z}_{m+1 \text { times }},(1,0, \ldots, 0))$, where $(1,0, \ldots, 0) \in Z^{m+1}$ and $Z \times_{\text {lex }} \ldots \times_{\text {lex }} Z$ is the lexicographic product of $Z m+1$ times.

Proposition 5.5. [[13] 1) $S_{1}^{\omega(m)}$ is generated by $m$ generators: $(0, \ldots, 0,1), \ldots,(0,1,0, \ldots, 0)$;

2) $S_{1}^{\omega(k)}$ is a homomorphic image of $S_{1}^{\omega(m)}$ for $k \leq m$.

3) 1-generated free $S_{1}^{\omega}$-algebra $F_{\mathcal{V}\left(S_{1}^{\omega}\right)}(1)$ is isomorphic to $\left(S_{1}^{\omega}\right)^{2}$ with free generator $g=((0,1),(1,-1))$.

For the sake of simplicity, let us introduce the following notations for the generating elements of the algebra $S_{1}^{\omega(m)}(m \geq 2): c_{1}=(0,0, \ldots, 0,1), c_{2}=(0,0, \ldots, 1,0), \ldots, c_{m}=(0,1, \ldots, 0,0)$. Notice, that $S_{1}^{\omega}$-algebra $S_{1}^{\omega(2)}$ is generated by two generators $c_{1}=(0,0,1)$ and $c_{2}=(0,1,0)$.

Proposition 5.6. [16] 2-generated free $S_{1}^{\omega}$-algebra $F_{\mathcal{V}\left(S_{1}^{\omega}\right)}(2)$ is isomorphic to $\left(\operatorname{Rad}^{*}\left(\left(S_{1}^{\omega(2)}\right)^{2}\right)\right)^{2^{2}}$ with free generators $g_{1}=\left(\left(c_{1}, c_{2}\right), \neg\left(c_{1}, c_{2}\right),\left(c_{1}, c_{2}\right), \neg\left(c_{1}, c_{2}\right)\right)$ and $g_{2}=\left(\left(c_{2}, c_{1}\right),\left(c_{2}, c_{1}\right), \neg\left(c_{2}, c_{1}\right), \neg\left(c_{2}, c_{1}\right)\right)$.

\section{Unification problem}

Let $E$ be an equational theory. The $E$-unification problem is: given two terms $s, t$ (built from function symbols and variables), to find a unifier for them, that is, a uniform replacement of the variables occurring in $s$ and $t$ by other terms that make $s$ and $t$ equal by modulo $E$. For detailed information on the unification problem, we refer the readers to [27, $[28,[26]$.

Let us be more precise. Let $\mathcal{F}$ be a set of functional symbols and let $V$ be a set of variables. Let $T_{\mathcal{F}}(V)$ be the term algebra built from $\mathcal{F}$ and $V$, and $T_{\mathcal{F}_{m}}(V)$ be the term algebra of $m$-variable terms. Let $E$ be a set of identities of type $p\left(x_{1}, \ldots, x_{m}\right)=q\left(x_{1}, \ldots, x_{m}\right)$, where $p, q \in T_{\mathcal{F}_{m}}(V)$.

Let $\mathbf{V}$ be the variety of algebras over $\mathcal{F}$ axiomatized by the equations from $E$.

$A$ unification problem modulo $E$ is a finite set of pairs

$$
\mathcal{E}=\left\{\left(s_{j}, t_{j}\right): s_{j}, t_{j} \in T_{\mathcal{F}_{m}}(V), j \in J\right\},
$$

for some finite set $J$. A solution to (or a unifier for) $\mathcal{E}$ is a substitution (or an endomorphism of the term algebra $\left.T_{\mathcal{F}_{m}}(V)\right) \sigma$ (which is extension of the map $s: V_{m} \rightarrow T_{\mathcal{F}_{m}}(V)$, where $V_{m}\left(=\left\{x_{1}, \ldots, x_{m}\right\}\right.$ ) is the set 
of $m$ variables) such that the identity $\sigma\left(s_{j}\right)=\sigma\left(t_{j}\right)$ holds in every algebra of the variety $\mathbf{V}$. The problem $\mathcal{E}$ is solvable (or unifiable) if it admits at least one unifier.

Let $(X, \preceq)$ be a quasi-ordered set (i. e. $\preceq$ is a reflexive and transitive relation). A $\mu$-set [28] for $(X, \preceq)$ is a subset $M \subseteq X$ such that: (1) every $x \in X$ is less or equal to some $m \in M$; (2) all elements of $M$ are mutually $\preceq$-incomparable. There might be no $\mu$-set for $(X, \preceq)$ (in this case we say that $(X, \preceq)$ has type 0 ) or there might be many of them, due to the lack of antisymmetry. However, all $\mu$-sets for $(X, \preceq)$, if any, must have the same cardinality. We say that $(X, \preceq)$ has type $1, \omega, \infty$ iff it has a $\mu$-set of cardinality 1 , of finite (greater than 1) cardinality or of infinite cardinality, respectively.

Substitutions are compared by instantiation in the following way: we say that $\left.\sigma: T_{\mathcal{F}_{m}}(V) \rightarrow T_{\mathcal{F}_{m}}(V)\right)$ is more general than $\tau: T_{\mathcal{F}_{m}}(V) \rightarrow T_{\mathcal{F}_{m}}(V)$ (written as $\tau \preceq \sigma$ ) iff there is a substitution $\eta: T_{\mathcal{F}_{m}}(V) \rightarrow T_{\mathcal{F}_{m}}(V)$ such that for all $x \in V_{m}$ we have $E \vdash \eta(\sigma(x))=\tau(x)$. The relation $\preceq$ is quasi-order.

Let $U_{E}(\mathcal{E})$ be the set of unifiers for the unification problem $\mathcal{E}$; then $\left(U_{E}(\mathcal{E}), \preceq\right)$ is a quasi-ordered set.

We say that an equational theory $E$ has:

1. Unification type 1 iff for every solvable unification problem $\mathcal{E}, U_{E}(\mathcal{E})$ has type 1 ;

2. Unification type $\omega$ iff for every solvable unification problem $\mathcal{E}, U_{E}(\mathcal{E})$ has type $\omega$;

3. Unification type $\infty$ iff for every solvable unification problem $\mathcal{E}, U_{E}(\mathcal{E})$ has type 1 or $\omega$ or $\infty$ - and there is a solvable unification problem $\mathcal{E}$ such that $U_{E}(\mathcal{E})$ has type $\infty$;

4. Unification type nullary, if none of the preceding cases applies.

Following Ghilardi [27], who has introduced the relevant definitions for $E$-unification from an algebraic point of view, by an algebraic unification problem we mean a finitely presented algebra $A$ of $\mathbf{V}$. In this context, an $E$-unification problem is simply a finitely presented algebra $A$, and a solution for it (also called a unifier for $A$ ) is a pair given by a projective algebra $P$ and a homomorphism $u: A \rightarrow P$. The set of unifiers for $A$ is denoted by $U_{E}(A)$. $A$ is said to be unifiable or solvable iff $U_{E}(A)$ is not empty. Given another algebraic unifier $w: A \rightarrow Q$, we say that $u$ is more general than $w$, written $w \preceq u$, if there is a homomorphism $g: P \rightarrow Q$ such that $w=g u$.

The set of all algebraic unifiers $U_{E}(A)$ of a finitely presented algebra $A$ forms a quasi-ordered set with the quasi-ordering $\preceq$.

The algebraic unification type of an algebraically unifiable finitely presented algebra $A$ in the variety $\mathbf{V}$ is now defined exactly as in the symbolic case, using the quasi-ordering set $\left(U_{E}(A), \preceq\right)$. If $m$-generated finitely presented algebra of an equational class $\mathbf{V}$ is projective, then $I d_{A}$ will be most general unifier for A.

Proposition 6.1. [14] The unification type of the equational class $\mathcal{V}\left(S_{1}^{\omega}\right)$ is 1, i. e. unitary.

\section{$7 \quad$ Monadic $M V$-algebras}

The finitely valued propositional calculi, which have been described by Łukasiewicz and Tarski in [33.] are extended to the corresponding predicate calculi. The predicate Łukasiewicz (infinitely valued) logic $Q L$ is defined in the following standard way. The existential (universal) quantifier is interpreted as supremum (infimum) in a complete $M V$-algebra. Then the valid formulas of predicate calculus are defined as all formulas having value 1 for any assignment. The functional description of the predicate calculus is given by Rutledge in [3.9]. Scarpellini in [40] has proved that the set of valid formulas is not recursively enumerable. Monadic $M V$-algebras were introduced and studied by Rutledge in [39] as an algebraic model for the predicate calculus $Q L$ of Łukasiewicz infinite-valued logic, in which only a single individual variable occurs. Rutledge followed P.R. Halmos' study of monadic Boolean algebras. In view of the incompleteness of the predicate calculus, the result of Rutledge in [3.9], showing the completeness of the monadic predicate calculus, has been of great interest.

Let $L$ denote a first-order language based on $\cdot,+, \rightarrow, \neg, \exists$ and let $L_{m}$ denote a propositional language based on $\cdot,+, \rightarrow, \neg, \exists$. Let $\operatorname{Form}(L)$ and $\operatorname{Form}\left(L_{m}\right)$ be the set of all formulas of $L$ and $L_{m}$, respectively. We fix a variable $x$ in $L$, associate with each propositional letter $p$ in $L_{m}$ a unique monadic predicate $p^{*}(x)$ in $L$ and define by induction a translation $\Psi: \operatorname{Form}\left(L_{m}\right) \rightarrow \operatorname{Form}(L)$ by putting: 
- $\Psi(p)=p^{*}(x)$ if $p$ is propositional variable,

- $\Psi(\alpha \circ \beta)=\Psi(\alpha) \circ \Psi(\beta)$, where $\circ=\cdot,+, \rightarrow$,

- $\Psi(\exists \alpha)=\exists x \Psi(\alpha)$.

Through this translation $\Psi$, we can identify the formulas of $L_{m}$ with monadic formulas of $L$ containing the variable $x$.

An algebra $A=(A, \oplus, \odot, \neg, \exists, 0,1)$ is said to be a monadic $M V$-algebra ( $M M V$-algebra for short) if $A=(A, \oplus, \odot, \neg, 0,1)$ is an $M V$-algebra and in addition $\exists$ satisfies the following identities:

E1. $x \leq \exists x$,

E2. $\exists(x \vee y)=\exists x \vee \exists y$,

E3. $\exists \neg(\exists x)=\neg(\exists x)$,

E4. $\exists(\exists x \oplus \exists y)=\exists x \oplus \exists y$,

E5. $\exists(x \odot x)=\exists x \odot \exists x$,

E6. $\exists(x \oplus x)=\exists x \oplus \exists x$.

A subalgebra $A_{0}$ of an $M V$-algebra $A$ is said to be relatively complete if for every $a \in A$ the set $\left\{b \in A_{0}: a \leq b\right\}$ has a least element.

Let $(A, \oplus, \odot, \neg, \exists, 0,1)$ be a monadic $M V$-algebra. By [3.], the $M V$-algebra $\exists A(=\{\exists a: a \in A\})$ is a relatively complete subalgebra of the $M V$-algebra $(A, \oplus, \odot, \neg, 0,1)$, and $\exists a=\inf \{b \in \exists A: a \leq b\}$.

A subalgebra $A_{0}$ of an $M V$-algebra $A$ is said to be m-relatively complete [II], if $A_{0}$ is relatively complete and two additional conditions hold:

(\#) $(\forall a \in A)\left(\forall x \in A_{0}\right)\left(\exists v \in A_{0}\right)(x \geq a \odot a \Rightarrow v \geq a \& v \odot v \leq x)$,

(\#\#) $(\forall a \in A)\left(\forall x \in A_{0}\right)\left(\exists v \in A_{0}\right)(x \geq a \oplus a \Rightarrow v \geq a \& v \oplus v \leq x)$.

Proposition 7.1. [ए2] Let $(A, \oplus, \odot, \neg, \exists, 0,1)$ be a monadic $M V$-algebra. Then the $M V$-subalgebra $\exists A$ of $M V$-algebra $(A, \oplus, \odot, \neg, 0,1)$ is $m$-relatively complete.

Proposition 7.2. [[2] There exists a one-to-one correspondence between:

(1) monadic $M V$-algebras $(A, \oplus, \odot, \neg, \exists, 0,1)$;

(2) the pairs $\left(A, A_{0}\right)$, where $A_{0}$ is m-relatively complete subalgebra of $A$;

(3) the pairs $\left(A, A_{0}\right)$, where $A_{0}$ is a subalgebra of $A$ and the canonical embedding $h: A_{0} \hookrightarrow A$ has left m-adjoint function.

\subsection{Multimodal epistemic Łukasiewicz logic with application in immune system}

In [17] we extend 3-valued Łukasiewicz logic $\mathrm{L}_{3}$ to the 3-valued multimodal Łukasiewicz logic $E \mathrm{Ł}_{3}(n)$ by adding $n$ unary modal "knowledge" operators $\square_{i}$ and $\diamond_{i}(i=1, \ldots, n)$ to the language of $\mathrm{E}$. We extend 3-valued multimodal Lukasiewicz logic $E \mathrm{E}_{3}(n)$ to the logic $E \mathrm{E}_{3}^{\square}(n)$ by adding global modal (knowledge) operators $\square$ and $\diamond$ to the language of $E \mathrm{E}_{3}(n)$.

The knowledge operators model a community of ideal knowledge agents who have the properties of veridical knowledge (everything they know is true), fuzzy knowledge (everything they know is quasitrue, positive introspection (they know what they know) and negative introspection (they know what they do not know) and so on. The knowledge operators permit the following interpretation:

$\square_{i} \alpha-" i$ knows proposition $\alpha "$;

$\diamond_{i} \alpha-" i$ does not know that proposition $\alpha$ is false".

A 3-valued Kripke frame for agent $i$ is a pair $\mathfrak{J}_{\mathfrak{i}}=\left(W_{i}, R_{i}\right), i=1, \ldots, n$, consisting of a non-empty set $W_{i}$ of elements called the states of the agent $i$ (or possible worlds of the agent $i$ ); $R_{i} \subset W_{i} \times W_{i}$ is a binary reflexive and transitive relation on $W_{i}$ (called the accessibility relation for agent $i$ ). 
A 3-valued Kripke model for agent $i$ (or simply, Kripke model for agent $i$, when there is no ambiguity) is a pair $\mathfrak{M}_{i}=\left(\mathfrak{J}_{i}, e_{i}\right), i=1, \ldots, n$, where $\mathfrak{J}_{\mathfrak{i}}=\left(W_{i}, R_{i}\right)$ is Kripke frame for agent $i$ and $e_{i}: \operatorname{Var} \times W_{i} \rightarrow S_{2}$ is a function, called evaluation for agent $i$, which maps every propositional variable $p \in \operatorname{Var}$ and possible world $w \in W_{i}$ to the set of truth values $S_{2}, i=1, \ldots, n$, such that if $e_{i}(p, w)=1$ and $\left(w, w^{\prime}\right) \in R_{i}$ then $e_{i}\left(p, w^{\prime}\right)=1$. If $\varphi$ is a propositional formula of $\mathrm{E}_{3}$, then $e_{i}(\varphi, w) \in S_{2}$ is a propositional evaluation for agent $i$; if $\varphi$ is a modal formula, then $e_{i}\left(\diamond_{i} \varphi, w\right)=\bigvee\left\{e_{i}\left(\varphi, w^{\prime}\right):\left(w, w^{\prime}\right) \in R_{i}\right\} ; e_{i}\left(\square_{i} \varphi, w\right)=\bigwedge\left\{e_{i}\left(\varphi, w^{\prime}\right):\left(w, w^{\prime}\right) \in R_{i}\right\}$ for every $w \in W_{i}, i=1, \ldots, n$.

A modal formula $\varphi$ is said to be modally valid for agent $i$ when it is evaluated to 1 in all Kripke models for agent $i$; it is said to be modally 1-satisfiable for agent $i$ when there is some Kripke model for agent $i$ and some world $w$ such that $e_{i}(\varphi, w)=1$; and it is said to be modally satisfiable for agent $i$ when it is 1-satisfiable for agent $i$. A modal formula $\varphi$ is said to be modally valid when it is evaluated to 1 in all Kripke models for every agent $i$.

A 3-valued descriptive Kripke frame is a pair $\mathfrak{J}=(W, R), W=\left\{W_{1}, \ldots, W_{n}\right\}$ is the set of $n$ agents (or possible worlds); $R \subset W \times W$ is a binary reflexive and transitive relation on $W$ (called the accessibility relation between agents $i\left(=W_{i}\right)$ ).

A 3-valued descriptive Kripke global model (or descriptive Kripke global model) is a triple $\mathfrak{M}=(W, R, V)$ where $W=\left\{W_{1}, \ldots, W_{n}\right\}$ is the set of $n$ agents (or possible worlds); $R \subset W \times W$ is a binary relation on $W$ (called the accessibility relation between agents $\left.i\left(=W_{i}\right)\right) ; V\left(\varphi, W_{i}\right)=\bigwedge\left\{e_{i}(\varphi, w): w \in W_{i}, e_{i}: \operatorname{Var} \times W_{i} \rightarrow\right.$ $\left.S_{2}\right\}, V\left(\square \varphi, W_{i}\right)=\bigwedge\left\{V\left(\varphi, W_{j}\right):\left(W_{i}, W_{j}\right) \in R\right\}, V\left(\diamond \varphi, W_{i}\right)=\bigvee\left\{V\left(\varphi, W_{j}\right):\left(W_{i}, W_{j}\right) \in R\right\}$.

A modal formula $\varphi$ is said to be globally modally valid when it is evaluated to 1 in all Kripke models for every agent $i \in\{1, \ldots, n\}$; it is said to be modally satisfiable when it is 1-satisfiable for some agent $i \in\{1, \ldots, n\}$.

We extend the language of $E \mathrm{E}_{3}(n)$ by two unary modal operators $\diamond$ and $\square$. A modal formula $\varphi$ is said to be globally modally valid when it is evaluated to 1 in all descriptive Kripke global models; it is said to be globally modally 1-satisfiable when 1-satisfiable for any agent $i \in\{1, \ldots, n\}$; and it is said to be globally modally satisfiable when it is 1-satisfiable for all descriptive Kripke models.

The logic $E \mathrm{E}_{3}(n)$ is defined as the set of its modal formulas that are modally valid. It is worth pointing out that for this modal logic the modal operators are interdefinable by means of the modally valid formulas $\diamond_{i} \varphi \leftrightarrow \neg \square_{i} \neg \varphi$ and $\square_{i} \varphi \leftrightarrow \neg \diamond_{i} \neg \varphi$.

The logic $E \mathrm{E}_{3}^{\square}(n)$, the language of which is enriched of the language of $E \mathrm{E}_{3}$ by two unary modal operators $\diamond$ and $\square$, is defined as the set of its modal formulas that are globally modally valid. From the definition of the descriptive Kripke frame we can deduce that the formulas $\square \varphi \leftrightarrow \square_{1} \varphi \wedge \ldots \wedge \square_{n} \varphi$ and $\diamond \varphi \leftrightarrow \diamond_{1} \varphi \vee \ldots \vee \diamond_{n} \varphi$ are globally modally valid.

[17] has studied 3-valued multimodal Lukasiewicz logic and its semantic - descriptive Kripke models with respect of which this logic is complete and using the ones for representation of immune systems. We can understand the set of $T$-cells as the set of agents. In many ways the immune system is a black box; although many of its inputs and outputs are known, exactly how the system achieves its function is the subject of many investigations. Laboratory experiments provide large quantities of data, allowing components (agents ( $T$-cells), state of the agents $(T$-cells $))$ within the black box to be identified, but there remain many details of how the components (agents $(T$-cells), state of the agents $(T$-cells)) of the system carry out their functions, or on the nature of the interaction between components. There are many variables in such systems that exhaustive testing to establish these details is not feasible. Multimodal logic and its corresponding Kripke model is ideally suited to describing immunological systems at this level: they may be represented as a relational system of interacting elements (components), where the components themselves may have complex, non-deterministic, individual behavior. Moreover, use of multimodal logic and Kripke model gives access to a range of investigatory techniques, including simulation, verification via logical properties.

We suggest the following schemata of axioms for $E \mathrm{Ł}_{3}(n)$ : to the schemata of axioms of $\mathrm{七}_{3}$ we add

1) $\square_{i} \varphi \rightarrow \varphi, \quad i=1, \ldots, n$,

2) $\square_{i} \varphi \rightarrow \square_{i} \square_{i} \varphi, \quad i=1, \ldots, n$

3) $\square_{i}(\varphi \wedge \psi) \leftrightarrow\left(\square_{i} \varphi \wedge \square_{i} \psi\right), \quad i=1, \ldots, n$,

4) $\square_{i}(\varphi \& \varphi) \leftrightarrow\left(\square_{i} \varphi \& \square_{i} \varphi\right), \quad i=1, \ldots, n$,

5) $\square_{i}(\varphi \underline{\vee} \varphi) \leftrightarrow\left(\square_{i} \varphi \underline{\vee} \square_{i} \varphi\right), \quad i=1, \ldots, n$, 
6) $\diamond_{i} \varphi \rightarrow \square_{i} \diamond_{i} \varphi, i=1, \ldots, n$,

inference rules: $\varphi, \varphi \rightarrow \psi / \psi, \varphi / \square_{i} \varphi, i=1, \ldots, n$.

We suggest the following schemata of axioms for $E \mathrm{E}_{3}^{\square}(n)$ : to the schemata of axioms of $\mathrm{E}_{3}$ we add

1) $\square_{i} \varphi \rightarrow \varphi, \quad i=1, \ldots, n$,

2) $\square_{i} \varphi \rightarrow \square_{i} \square_{i} \varphi, \quad i=1, \ldots, n$,

3) $\square_{i}(\varphi \wedge \psi) \leftrightarrow\left(\square_{i} \varphi \wedge \square_{i} \psi\right), \quad i=1, \ldots, n$,

4) $\square_{i}(\varphi \& \varphi) \leftrightarrow\left(\square_{i} \varphi \& \square_{i} \varphi\right), \quad i=1, \ldots, n$,

5) $\square_{i}(\varphi \underline{\vee} \varphi) \leftrightarrow\left(\square_{i} \varphi \underline{\vee} \square_{i} \varphi\right), \quad i=1, \ldots, n$,

6) $\square \varphi \leftrightarrow \bigwedge_{i=1}^{n} \square_{i} \varphi, \quad i=1, \ldots, n$,

7) $\diamond_{i} \varphi \rightarrow \square_{i} \diamond_{i} \varphi, i=1, \ldots, n$,

8) $\square_{i}(\varphi \rightarrow \psi) \rightarrow\left(\square_{i} \varphi \rightarrow \square_{i} \psi\right), \quad i=1, \ldots, n$,

9) $\square(\varphi \rightarrow \psi) \rightarrow(\square \varphi \rightarrow \square \psi)$,

inference rules: $\varphi, \varphi \rightarrow \psi / \psi, \varphi / \square \varphi$.

Proposition 7.3. [I7] (Completeness of $\left.E E_{3}(n)\left(E E_{3}^{\square}(n)\right)\right)$ A formula $\varphi$ is (globally) modally valid formula of $\left.E E_{3}(n)\left(E E_{3}^{\square}(n)\right)\right)$ iff it is a theorem of $\left.E E_{3}(n)\left(E E_{3}^{\square}(n)\right)\right)$.

\section{Dynamic $M V$-algebras}

Dynamic logic is a formal system for reasoning about programs. Dynamic logic is a modal logic for reasoning about dynamic behavior taking into account a discrete time. Dynamic logic is an extension of modal logic originally intended for reasoning about computer programs and later applied to more general complex behaviors arising in linguistics, philosophy, AI, and other fields.

Modal logic is characterized by the modal operators $\square p$ asserting that $p$ is necessarily the case, and $\diamond p$ asserting that $p$ is possibly the case. Dynamic logic extends this by associating to every action $a$ the modal operators $[a]$ and $\langle a\rangle$, thereby making it a multimodal logic. The meaning of $[a] p$ is that after performing action $a$ it is necessarily the case that $p$ holds, that is, $a$ must bring about $p$. The meaning of $\langle a\rangle p$ is that after performing $a$ it is possible that $p$, that is, $a$ might bring about $p$. These operators are related by $[a] p \equiv \neg\langle a\rangle \neg p$ and $\langle a\rangle p \equiv \neg[a] \neg p$, analogously to the relationship between the universal $\forall$ and existential $\exists$ quantifiers.

Dynamic logic permits compound actions built up from smaller actions. Given actions $a$ and $b$, the compound action $a \cup b$, choice, is performed by performing one of $a$ or $b$. The compound action $a ; b$, sequence, is performed by performing first $a$ and then $b$. The compound action $a^{*}$, iteration, is performed by performing $a$ zero or more times, sequentially. The constant action 0 or BLOCK does nothing and does not terminate, whereas the constant action 1 either SKIP or NOP, definable as $0^{*}$, does nothing but does terminate.

We have proposed the notion of a dynamic $M V$-algebra, which integrates an abstract notion of proposition with an equally abstract notion of action. Just as propositions tend to band together to form $M V$-algebras with operations $x \oplus y$, and $\neg x$, so do actions organize themselves into regular algebras, with operations $a \cup b, a ; b$, and $a^{*}$. Analogously to the proposition $p \underline{\vee} q$ being the strong disjunction (the algebraic counterpart of which is $x \oplus y), p \vee q$ being the disjunction of propositions $p$ and $q$, and $\neg p$ the negation of $p$, the action $a \cup b$ is the choice of actions $a$ or $b, a ; b$, or just $a b$, is the sequence $a$ followed by $b$, and $a^{*}$ is the iteration of a indefinitely often.

It is natural to think of an action as being able to bring about a proposition. We write $\langle a\rangle p$, or just ap, pronounced "a enables $p "$, as the proposition that action $a$ can bring about proposition $p$. A dynamic algebra then is a $M V$-algebra $(A, \oplus, \odot, \neg, 0,1)$, a regular algebra $\left(R, \cup, ;,{ }^{*}\right)$, and the enables operation $\diamond: R \times A \rightarrow A$.

Dynamic propositional Łukasiewicz logic $D P €$ is designed for representing and reasoning about propositional Łukasiewicz properties of programs. Its syntax is based upon two sets of symbols: a countable set $\operatorname{Var}\left(=p, p_{1}, p_{2}, \ldots, q, q_{1}, q_{2},\right)$ of propositional variables and a countable set $\Pi$ of atomic programs. So the language $\mathcal{L}$ of $D P €$ is given by a countable set Var of propositional variables and a countable set $\Pi$ of 
atomic programs. Formulas and programs $F P(\mathcal{L})$, which we name formulas, over this base are defined as follows:

- Every propositional variable is a formula;

- $\perp$ (false $)$ is a formula;

- If $\varphi$ is a formula then $\neg \varphi(\operatorname{not} \varphi)$ is a formula;

- If $\varphi$ and $\psi$ are formulas then $(\phi \underline{\vee} \psi)$ ( $\underline{\vee}$ is a strong disjunction) is a formula;

- If $\varphi$ and $\psi$ are formulas then $(\varphi \& \psi)$ ( \& is a strong conjunction) is a formula;

- If $\varphi$ and $\psi$ are formulas then $(\varphi \vee \psi)(\varphi$ or $\psi)$ is a formula;

- If $a$ is a program and $\varphi$ is a formula then $[a] \varphi$ (every execution of $a$ from the present state leads to a state where $\varphi$ is true) is a formula

- Every atomic program is a program;

- If $a$ and $b$ are programs then $(a ; b)$ (do $a$ followed by $b$ ) is a program;

- If $a$ and $b$ are programs then $(a \cup b)$ (do $a$ or $b$, non-deterministically) is a program;

- If $a$ is a program then $a^{*}$ (repeat $a$ a finite, but non-deterministically determined, number of times) is a program;

- If $\varphi$ is a formula then $\varphi$ ? (proceed if $\varphi$ is true, else fail) is a program.

The other Eukasiewicz connectives $1, \rightarrow$ and $\leftrightarrow$ are used as abbreviations in the standard way ( $1 \equiv$ $\perp \underline{\vee} \neg \perp, p \rightarrow q \equiv \neg p \underline{\vee} q, p \leftrightarrow q \equiv(p \rightarrow q) \wedge(q \rightarrow p))$. In addition, we abbreviate $\neg[a] \neg \varphi$ to $\langle\varphi\rangle$ (some execution of $a$ from the present state leads to a state where $\varphi$ is true) as in modal logic. We write $a^{n}$ for $a ; \ldots ; a$ with $n$ occurrences of $a$. More formally:

- $a^{0}={ }_{d f} 1$ ?

- $a^{n+1}={ }_{d f} a ; a^{n}$.

Finally, we adopt the standard rules for omission of parentheses.

The axioms of DP€ are the axioms of Łukasiewicz logic (L1) - (L4):

(L1) $\varphi \rightarrow(\psi \rightarrow \varphi)$,

(L2) $(\varphi \rightarrow \psi) \rightarrow((\psi \rightarrow \chi) \rightarrow(\varphi \rightarrow \chi))$,

(L3) $(\neg \varphi \rightarrow \neg \psi) \rightarrow(\psi \rightarrow \varphi)$,

(L4) $((\varphi \rightarrow \psi) \rightarrow \psi) \rightarrow(\psi \rightarrow \varphi) \rightarrow \varphi)$.

plus the following schemata of modal parts. For any formulas $\varphi, \psi$ and and any programs $a, b$ :

$$
\begin{aligned}
& \text { Ax0 }[a](\varphi \rightarrow \psi) \rightarrow([a] \varphi \rightarrow[a] \psi), \\
& \text { Ax1 }[a ; b] \varphi \leftrightarrow[a][b] \varphi \\
& \text { Ax2 }[a \cup b] \varphi \leftrightarrow[a] \varphi \vee[b] \varphi, \\
& \text { Ax3 }\left[a^{*}\right] \varphi \leftrightarrow \varphi \wedge[a]\left[a^{*}\right] \varphi, \\
& \text { Ax4 }[\varphi ?] \psi \leftrightarrow \varphi \rightarrow \psi), \\
& \text { Ax5 }[a](\varphi \& \varphi) \leftrightarrow[a] \varphi \&[a] \varphi, \\
& \text { Ax6 }[a](\varphi \underline{\vee} \varphi) \leftrightarrow[a] \varphi \underline{\vee}[a] \varphi .
\end{aligned}
$$

and closed under the following rules of inference:

(MP) from $\varphi$ and $\varphi \rightarrow \psi$ infer $\psi$,

(N) from $\varphi$ infer $[a] \varphi$,

(I) $\varphi \rightarrow[a] \varphi$ infer $\varphi \rightarrow\left[a^{*}\right] \varphi$.

Notice that these axioms presented as multimodal Łukasiewicz logic [177].

The axioms of $D P \mathrm{E}_{n}$ are the axioms $D P €$ plus the following axioms:

$\left(L_{n} 5\right) \varphi^{n} \leftrightarrow \varphi^{n-1}$,

$\left(L_{n} 6\right) n\left(\varphi^{k}\right) \leftrightarrow\left(k\left(\varphi^{k-1}\right)\right)^{n}$,

for every integer $2 \leq k \leq n-2$ that does not divide $n-1$ [ [Z29].

Dynamic $M V$-algebra (dynamic $M V_{n}$-algebra, $\left.n \in Z^{+}\right)$combine $M V$-algebra $\left(M V_{n}\right.$-algebra) $\mathcal{M}=$ $(M, \oplus, \odot, \sim, 0,1)$ and regular algebra $\mathcal{R}=\left(R, \cup, ;{ }^{*}\right)$ into a single finitely axiomatized class $(\mathcal{M}, \mathcal{R}, \diamond)$ 
resembling an $R$-module with scalar multiplication $\diamond: R \times M \rightarrow M$. A dynamic $M V$-algebra (dynamic $M V_{n}$-algebra) $\mathcal{D}=(\mathcal{M}, \mathcal{R}, \diamond)$ satisfies the following axioms: for any $x, y \in M$ and $a, b \in R$

1. $\mathcal{M}$ is $M V$-algebra ( $M V_{n}$-algebra).

2. $a 0=0$.

3. $a(x \vee y)=a x \vee a y$.

4. $(a \cup b) x=a x \vee b x$.

5. $(a b) x=a(b x)$.

6. $a(x \oplus x)=a x \oplus a x$.

7. $a(x \odot x)=a x \odot a x$.

8. $x \vee a a^{*} x \leq a^{*} x \leq x \vee a^{*}(\neg x \wedge a x)$.

If in addition a dynamic $M V$-algebra satisfies the following condition

9. $x ? y=x \wedge y$,

then it is called test algebra.

Proposition 8.1. (Completeness theorem). A formula $\varphi$ of dynamic propositional logic DPE is a tautology iff it is a theorem of the logic.

\subsection{Dynamic Łukasiewicz Logic and its Application to Immune System}

The results obtained for dynamic Łukasiewicz logic have been applied for immune system in [1]] having another interpretation of modal operators. It is introduced an immune dynamic $n$-valued Łukasiewicz logic $I D \mathrm{E}_{n}$ on the base of $n$-valued Łukasiewicz logic $\mathrm{E}_{n}$ and corresponding to it immune dynamic $M V_{n}$-algebra ( $I D L_{n}$-algebra), $1<n<\omega$, which are algebraic counterparts of the logic, that in turn represent two-sorted algebras $(\mathcal{M}, \mathcal{R}, \diamond)$ that combine the varieties of $M V_{n}$-algebras $\mathcal{M}=(M, \oplus, \odot, \sim, 0,1)$ and regular algebras $\mathcal{R}=\left(R, \cup, ;,{ }^{*}\right)$ into a single finitely axiomatized variety resemblig $R$-module with "scalar" multiplication $\diamond$. Kripke semantics is developed for immune dynamic Eukasiewicz logic $I D \mathrm{E}_{n}$ with application in immune system.

Immune dynamic $n$-valued Lukasiewicz logic $I D \mathrm{E}_{n}$ is designed for representing and reasoning about propositional Łukasiewicz logic expected results (hypothesis) of (laboratory) experiments (results of experimentation). Its syntax is based upon two sets of symbols: a countable set $\operatorname{Var}\left(=\left\{p, p_{1}, p_{2}, \ldots, q, q_{1}, q_{2}, \ldots\right\}\right)$ of propositional variables and a countable set $\operatorname{Exp}(=\{a, b, c, \ldots\})$ of atomic experiments. So the language $\mathcal{L}$ of $I D \mathrm{~L}_{n}$ is given by a countable set Var of propositional variables and a countable set Exp of atomic experiments.

One of the semantics of $I D L_{n}$ are Kripke semantics [II8] that is similar to the ones that have been done in [[22]. In the presented case the formula $[a \cup b] \varphi$ means that whenever laboratory experiment $a$ or $b$ is successfully done, a state is reached where $\varphi$ holds, whereas the formula $\langle(a ; b)\rangle \varphi$ means that there is a sequence of consecutive laboratory experiments $a$ and $b$ such that a state is reached where $\varphi$ holds. Semantically speaking, formulas are interpreted by states and experiments are interpreted by binary relations over states in a Kripke model.

\section{THE EPILOGUE}

This paper is submitted for a Special Issue Dedicated to Prof. Antonio Di Nola. In this paper, I have taken responsibility to present the longtime friendship and joint scientific collaboration of two mathematicians: Antonio Di Nola and Revaz Grigolia, in the field of logical algebraic structures, that devoted to the 75th birthday of Prof. Antonio Di Nola. I express my deep gratitude to Antonio Di Nola for this longtime collaboration and friendship and Journal of Algebraic Hyperstructures and Logical Algebras for presented to me passibility to express this gratitude frankly.

\section{References}

[1] S. Aguzzoli, S. Bova, V. Marra, Applications of finite duality to locally finite varieties of BL-algebras, in Logical Foundations of Computer Science. (International Symposium, LFCS 2009, Deerfield Beach, 
FL, USA), ser. Lecture Notes in Computer Science, S. Artemov and A. Nerode, Eds. Berlin: Springer, 5407, (2009), 1-15.

[2] L.P. Belluce, C.C. Chang, A weak completeness theorem for infinite valued predicate logic, Journal of Symbolic Logic, 28 (1963), 43-50.

[3] L.P. Belluce, A. Di Nola, B. Gerla, Perfect $M V$-algebras and their logic, Applied Categorical Structures, 15(1-2) (2007), 135-151.

[4] L.P. Belluse, A. Di Nola, A. Lettieri, Local MV-algebras, Rendiconti Circolo Matematico di Palermo, 42 (1993), 347-361.

[5] G. Birkhoff, Lattice theory, Providence, Rhode Island, 1967.

[6] L.M. Cabrer, D. Mundici, Projective MV-algebras and rational polyhedra, Algebra Universalis, 62 (2009), 63-74.

[7] C.C. Chang, Algebraic analysis of many-valued logics, Transactions of the American Mathematical Society, 88 (1958), 467-490.

[8] C.C. Chang, A new proof of the completeness of the Eukasiewicz axioms, Transactions of the American Mathematical Society, 93 (1959), 74-80.

[9] R.L.O. Cignoli, I.M.L. D’Ottaviano, D. Mundici, Algebraic foundations of many-valued reasoning, volume 7 of Trends in Logic Studia Logica Library. Kluwer Academic Publishers, Dordrecht, 2000.

[10] A. Di Nola, Representation and reticulation by quotients of $M V$-algebras, Ricerche di Matematica, (Naplies), 40 (1991), 291-297.

[11] A. Di Nola, R. Grigolia, Projective MV-algebras and their automorphism groups, Journal of MultiValued Logic and Soft Computing, 9 (2003), 291-317.

[12] A. Di Nola, R. Grigolia, On monadic MV-algebras, Annals of Pure and Applied Logic, 128(1-3) (2004), 125-139.

[13] A. Di Nola, R. Grigolia, Gödel spaces and perfect MV-algebras, Journal of Applied Logic, 13(3) (2015), $270-284$.

[14] A. Di Nola, R. Grigolia, G. Lenzi, Structural completeness and unification problem of the logic of chang algebra, Azerbaijan Journal of Mathematics, 6(1) (2016), 23-38.

[15] A. Di Nola, R. Grigolia, A. Lettieri, Projective MV-algebras, International Journal of Approximate Reasoning, 47 (2008), 323-332.

[16] A. Di Nola, R. Grigolia, R. Liparteliani, On the free $S_{1}^{\omega}$-algebras, Journal of Algebraic Hyperstructures and Logical Algebras, 1(2) (2020), 1-7.

[17] A. Di Nola, R. Grigolia, N. Mitskevich, Multimodal epistemic Eukasiewicz logics with application in immune system, Soft Computing, 19(11) (2015), 3341-3351.

[18] A. Di Nola, R. Grigolia, N. Mitskevich, G. Vitale, Dynamic Eukasiewicz logic and its application to immune system, Soft Computing, 25 (2021), 9773-9780.

[19] A. Di Nola, R. Grigolia, G. Panti, Finitely generated free MV-algebras and their automorphism groups, Studia Logica, 61(1) (1998), 65-78.

[20] A. Di Nola, R. Grigolia, L. Spada, A discrete representation of free MV-algebras, Mathematical Logic Quarterly, 56(3) (2010), 279-288.

[21] A. Di Nola, R. Grigolia, E. Turunen, Fuzzy logic of quasi-truth: An algebraic treatment, Springer, Series: Studies in Fuzziness and Soft Computing, Vol. 338, 117 pages, 2016. 
[22] A. Di Nola, R. Grigolia, G. Vitale, Dynamic Eukasiewicz logic and dynamic MV-algebras, International Journal of Approximate Reasoning, 124 (2020), 103-110.

[23] A. Di Nola, A. Lettieri, Perfect $M V$-algebras categorically equivalent to Abelian -groups, Studia Logica: An International Journal for Symbolic Logic, 53(3) (1994), 417-432.

[24] A. Di Nola, A. Lettieri, Equational characterization of all varieties of MV-algebras, Journal of Algebra, 221 (1999), 463-474.

[25] W. Dzik, Unification in some substructural logics of BL-algebras and hoops, Reports in Mathematical Logic, 43 (2008), 73-83.

[26] S. Ghilardi, Unification through projectivity, Journal of Logic and Computation, 7 (1997), 733-752.

[27] S. Ghilardi, Unification in intuitionistic and De Morgan logic, Journal of Symbolic Logic, 64(2) (1999), 859-880.

[28] S. Ghilardi, Best solving modal equations, Annals of Pure and Applied Logic, 102(3) (2000), 183-198.

[29] R. Grigolia, Algebraic analysis of Lukasiewicz-Tarski n-valued logical Systems, Proceedings of Tbilisi University, A 6-7 (149-150), (1973), 121-132 (in russian). [English translation: R. Grigolia, Algebraic analysis of Lukasiewicz-Tarski n-valued logical systems, Selected papers on Łukasiewicz Sentential Calculi, Wroclaw, (1977), 81-91].

[30] Y. Komori, Super-Lukasiewicz propositional logic, Nagoya Mathematical Journal, 84 (1981), 119-133.

[31] D. Kozen, A representation theorem for models of *-free PDL, Technical Report RC7864, IBM, September, 1979 .

[32] J. Łukasiewicz, O logice tròjwartósciowej, Ruch Filozoficzny, 5 (1920), 169 -171.

[33] J. Łukasiewicz, A. Tarski, Untersuchungen über den Aussagenkalkül, Comptes Rendus de la Société des Sciences et des Lettres de Varsovie, 23(iii) (1930), 30-50.

[34] V. Marra, L. Spada, Duality, projectivity, and unification in ukasiewicz logic and MV-algebras, Annals of Pure and Applied Logic, 164(3) (2013), 192-210.

[35] D. Mundici, Interpretation of AF $C^{*}$-algebras in Eukasiewicz sentential calculus, Journal of Functional Analysis, 65 (1986), 15-63.

[36] G. Panti, Varieties of MV-algebras, Journal of Applied Non-Classical Logics, 9(1) (1999), 141-157.

[37] E.L. Post, Introduction to a general theory of propositions, American Journal of Mathematics, 43 (1921), $163-185$.

[38] V.R. Pratt, Dynamic algebras and the nature of induction, in: 12th ACM Symposium on Theory of Computation, Los Angeles, (1980), 22-28.

[39] J.D. Rutledge, A preliminary investigation of the infinitely many-valued predicate calculus, Ph.D. Thesis, Cornell University, 1959.

[40] B. Scarpellini, Die nichaxiomatisierbarkeit des unendlichwertigen Pradikatenkalkulus von Eukasiewicz, Journal of Symbolic Logic, 27 (1962), 159-170.

[41] K. Segerberg, A completeness theorem in the modal logic of programs, Notices of the American Mathematical Society, 24(6) (1977), A-552. 